\title{
Design and implementation of the visualization tourism help system Based on the android
}

\author{
Yan Wang ${ }^{1, a}$, Wang $\mathrm{CJ}^{2, \mathrm{~b}}$, Yang $\mathrm{HS}^{3, \mathrm{c}}$ \\ ${ }^{1}$ Author is with the school of education, Hangzhou Normal University, Hangzhou, China \\ ${ }^{2}$ Author is with the school of education, Hangzhou Normal University, Hangzhou, China \\ ${ }^{3}$ Author (corresponding author) is with the school of education, Hangzhou Normal University, \\ Hangzhou, China \\ aemail:445217698@qq.com, bemail:1173402448@qq.com, cemail:hzjyhs@163.com
}

Keywords: Visualization; Instant Messaging; Information Push;The Silk Road

\begin{abstract}
According to the problem that the intelligence is not high of the soccer robot, using the mechanical theory as a guide, making some mechanical analyses and calculations on the pressure and transmutation states of chip kick mechanics, and conducting optimal design too, then making the structure of chip kick mechanics more and more rationalization. Experiments show that the new soccer robot controller features a quick response and high servo rigidity, and provide a kind of method for improving and perfecting the soccer robot control system, at the same time, filling the needs of producing.
\end{abstract}

\section{Introduction}

On June 22, 2014, the Silk Road properties for success, this article of economic artery that bearing the history of ancient civilization once again attracted the attention of the world, this is bound to attract a lot of people to retrace the Silk Road. As the last paragraph within the territory of China, the period of ancient "silk road" in Xinjiang was traffic arteries, there brought together many countries economic, political, and cultural exchange activities, forming of the unique features and various ethnic cultures, colorful costumes, beautiful music, hospitable people attract tourists from all over the world. But because of Xinjiang is located in the northwest where lack of resource and living scattered, how to improve the tourism experience of retracing the Silk Road is an urgent problem to be solved. With the rapidly development of mobile Internet, There are much wider stage to display based on the mobile terminal of service application development [1]. Therefore, it is great significance to develop a system based on mobile of the Silk Road in Xinjiang for help the tourism, such as providing guidance on line, pushing real-time information about accommodation catering , especially emergency help[2].

\section{The analysis of system requirements and the integrated design}

The Silk Road will attract a large number of visitors revisiting after its successful inscription. As an important part of ancient Silk Road, the Kumusi section will gather a great quantity of visitors, which generates a typical problem: visitors have some trouble in the way inevitably and seek help for others. However, Kumusi locates Northwest China and lacks of resources. People lives scattered. It is impossible to ask help in time. So, it is necessary to use the Internet. Although there are some Instant Messengers, for example QQ, they have a significant problem: the objects of help-seeking are undefined and obvious blindness. At the same time, it is hard to meet visitors' requirements of line and area services quickly. Visitors will not add the QQ numbers of service supplier to the address list or use other way to remember it before. Thus, we launch this system to afford better speedy and friendly services for visitors in Kumusi section and solve visitors' troubles.

This system aims at solving all kinds of visitors' requirements and promoting the efficient utilization of route guidance, accommodation, dining and emergency treatment resources. This 
service system can afford a real-time and visual help by virtues of a universal visual service platform [3].

The total design aim of the system is solving following problems: the difficult of objects of help—seeking undefined or call inconvenience when requester in trouble; the condition of difficult describe in real life[4]; the problem of lacking of humanization and friendly interaction in existing system; the problem of closure in service request system.

The system technique process includes: starting from user launching help information to accomplish assist and ending at mutual evaluate, and included the technique part of administrator supervising the whole system [5]. The specific system design chart shown in Figure1.

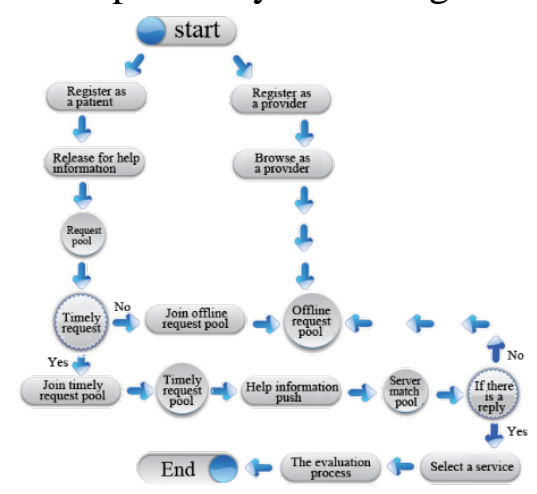

Fig.1. System technology flow chart

In technical process, we set up four middle pools: user request pool, instant request pool, offline request and server match pool. There are many different forms of requesting, such as multimedia and writing. User request pool used to record the request of users and facilitate the administrator's regulation. On this basis, we use labels to distinguish the request of instant and offline; it will not increase additional requests for memory.

Through pushing the requests of instant request pool to the service provider and users is labelled as specialist of this field, so that users can gather quick feedback. When service providers give the sign to provide services, users can accept the service by themselves. And then there is the evaluation process after services completed. If there is No Answer after a certain time, the request will automatically be converted to offline request pool and wait for the response.

Except for accepting push message, Service providers and users can also browse the request in offline request pool and make a response. The records of requests and responses will be loaded into the database, users and service providers can see it through the interface, users can also doing for maintenance.

After users' registration, information will be recorded in the database; the administrator can manage the users' data through the interface. For example: freezing the illegal accounts. Of course, users can access the database through the interface, to complete the management of personal information and good friends.

\section{The key technology}

Visualization of instant communication

This is the core technology of this system, the main content is divided into the following several aspects:

Video streaming collection: the Android platform support video recording but no native API can directly obtain video streaming. Local Socket can be used in the Android to correspond with Java and $\mathrm{c}++$, so that you can use Local Socket to intercept camera video streams, but this video streaming is not encoded, the amount of data is too large. So it is necessary to encode video after gather video streaming.

Video streaming coding: First, using the Media Recorder class programming to obtain the corresponding resolution H264 format video; By parsing 3GP files get the SPS/PPS information of H264 compressed video; Then through the Local Socket obtaining the local video data, 
subcontracting and determining the key frames of video streaming; Based on the judgment of key frames, deciding whether to send the SPS/PPS information.

Visualization of instant communication: Adopting the Live555 streaming media server to achieve real-time streaming video and broadcast in this system. Live555 is a cross-platform of c++ Open Source Program for providing streaming media solutions, it supports the standard streaming media transmission protocol, such as RTP/RTCP, RTSP, SIP. Specific implementation will also involve database operations and the native IP access operation, the main process as shown in figure2, after the client starts to get IP address of the machine and upload to the server, the server updates the database, and returns the target IP. The client then uploads video to the streaming media server, and attaches the target IP, while storing the streaming information of the streaming media server in the database. Server program transmit streaming to the target client according to the information database, and target IP.

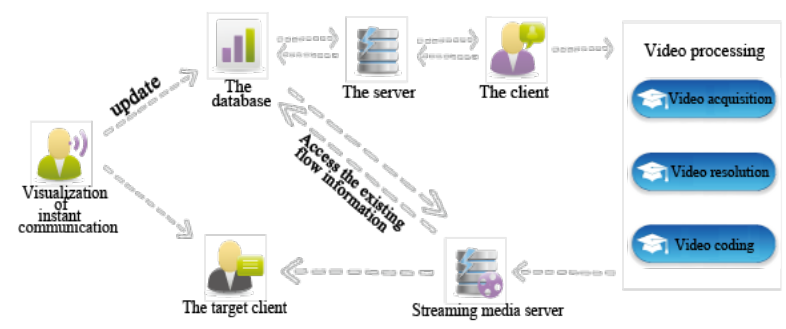

Fig.2.The flow chart of Visualization instant messaging technology

Information push service for help

VIP's help information will be pushed to the appropriate service provider, including Volunteers and third-party profit, etc. Faced with help information push, we developed a set of corresponding specialized push policy to solve the problem, including two modules of push the priority arrangement and Push object matching. Push the process as shown in fig3.

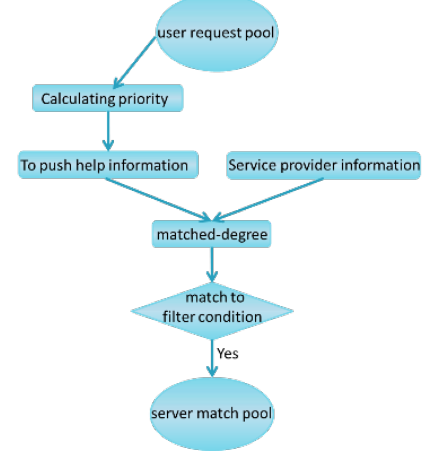

Fig.3.Push program of help information

When there is a new request, the system first insert user requests to the user requests pool, according to the request weight in descending order, sorting request queue by priority to push information one by one. System adopts dynamic priority, through the way of preemption priority to push for request processing.

When determining some help information for the candidate to push information, it's need to search service provider and extract the appropriate number of candidate service providers, to push for help information. To match candidate service providers and help information, it's need to calculate the similarity by using the weighted method. The choice of the standard for the candidate service provider is that the right value is greater than the threshold value, and push help information for the candidate service provider.

The implementation of user location

When helper issued corresponding request for help, we will conduct helpers' position in order to the server can serve the fast-site service. Similarly, we will also locate service providers' position so that the users can view their surrounding server [6].

With the help of the conventional map application API, we will conduct users' position. Based on system operation market, we will choose Baidu map API to get the latitude and longitude of the 
positioned person and helper. For the positioning information, we will ask the user for permission, if the user does not agree with position information, we will not be open [7]. If the user public location information, it can help server provide better service in a timely manner.

System design scheme for the client

The style of the mobile phone's APP design is to develop in the direction of minimalism and flattening, and through the survey, we found that such a design style is also acclaimed. When users need help, the minimalist style will greatly reduce the difficulty of their operations, make it easy to help and shorten time maximize.

And we start from the front-end interactive and carry on the corresponding system for mobile client front end design in order to reduce operation steps, simplify the operation interface and intuitive UI. All the work of the test programs are focusing on improving the quality of products. By preventing the various risks existing in the testing process, ultimately complete all the testing at a reasonable schedule control, and get the software that satisfied customers' requirements and expectations, maintainable and high quality. High-fidelity design drawings (part) as shown in figure4.

The code of system implementation

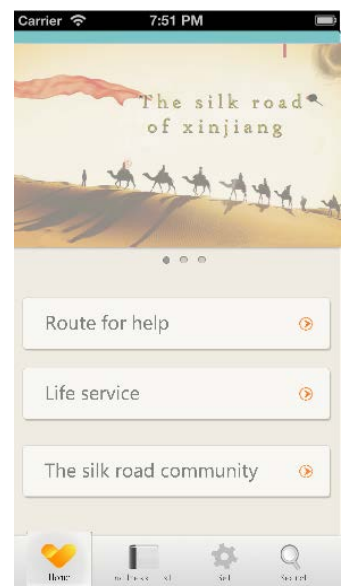

Fig.4.The client drawings

The main interface load layout files, and start other activity entry. The code is shown in figure5.

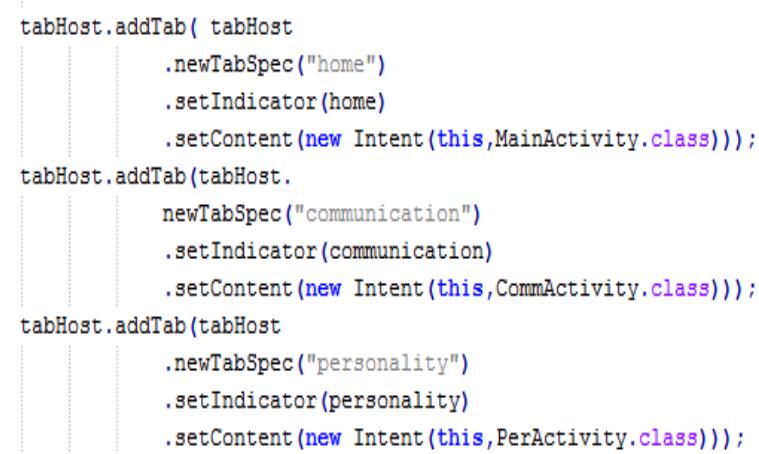

Fig.5.Loading layout file

The evaluation interface of the callers to the service is the pop-up box. The code is shown in figure6. 


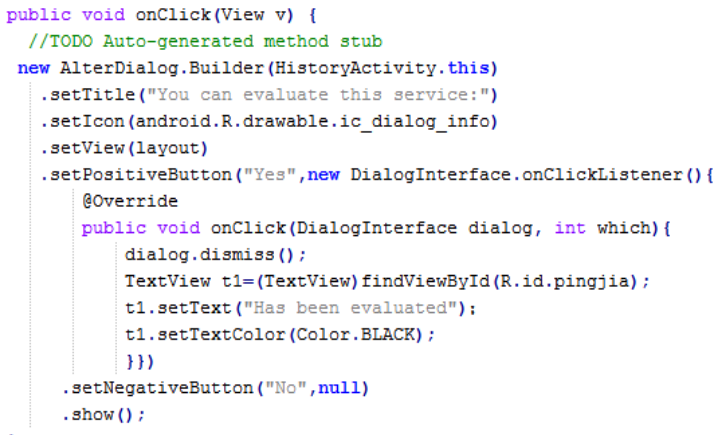

To get photos, the code is shown in figure7.

\section{Fig.6.Pop-up box}

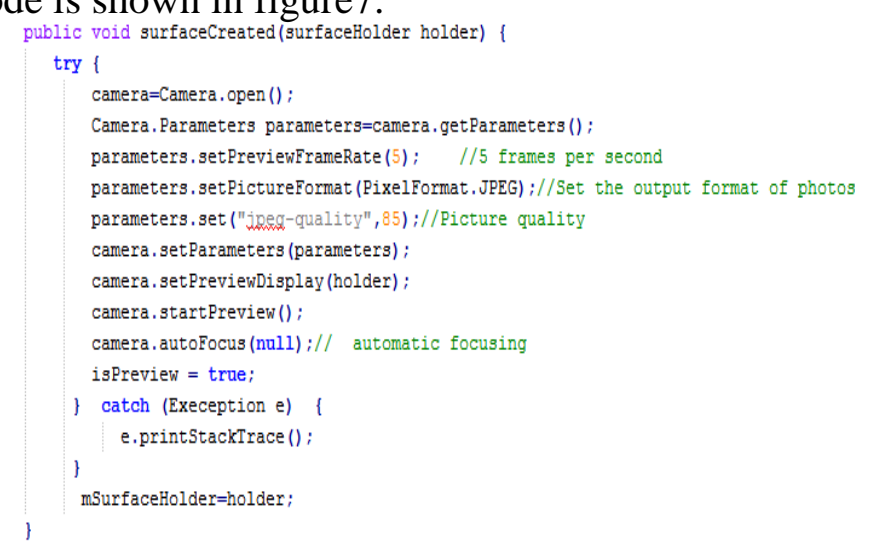

Fig.7.To get photos

Get video streaming data, and coding transmission. The code is shown in figure 8 . mVideoStartBtn. setonClickListener (new Button.onClicklistener()

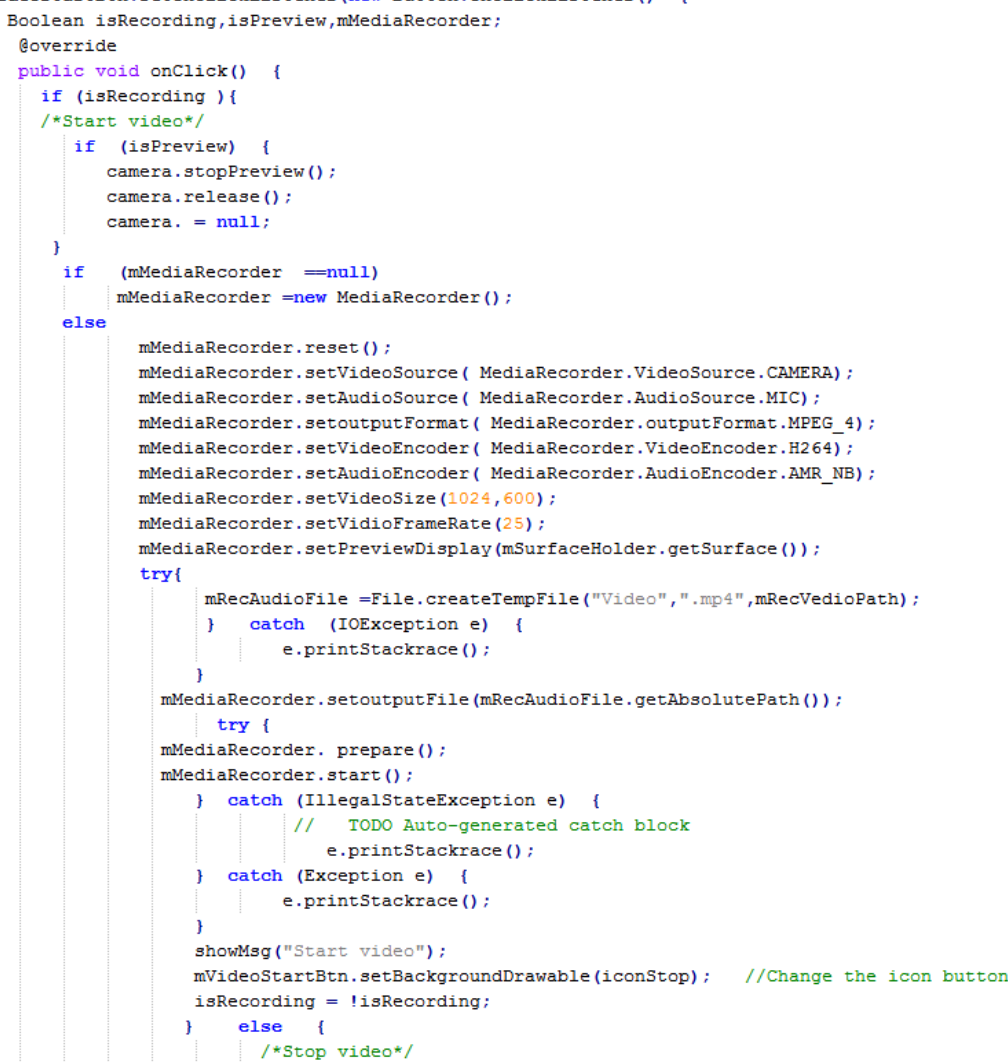

Fig.8.Video capture, encoding and transmission 


\section{Conclusion}

In the whole process of visualization help system, the system was tested through establishing rigorous testing model, and released the product that have high quality, can run and meet user needs at a reasonable development schedule.

The success of Silk Road properties will bring human heritage preservation more opportunities and challenges, and that will let more people understand the Silk Road and go into the Silk Road. The visual appeal system of Silk Road in Xinjiang is designed to help every retrace the Silk Road of Xinjiang and the people who live there. The system will be improved cognitive level of those who embrace the ancient ruins of ancient culture, provided diversified tourism appeal mechanism for those who need help, provided intelligent management solutions for the local tourist industry development, built communication platform for the people who know and don't familiar with the local culture and custom. Silk Road belongs to the large linear cultural heritages, the system expects to build a bridge between us and human cultural heritage of the Silk Road, in order to deepen the understanding and contact of the Silk Road of Xinjiang, and render to the transmission of cultural heritage, and solve the trouble as much as possible for every people who close to the Silk Road.

\section{Acknowledgement}

This research was key project of the National Social Science "The research of the cultural protection of the Silk Road and the Temporal and Spatial Visual Analysis Technique",No.12AZD118. The corresponding author of this paper is Huansong Yang.

\section{References}

[1] Dai Er-zheng. The design and implementation of device and video based on the Android [D]. Zhejiang University of technology, 2012.

[2] Shen Wen-jie. The realization of the intelligent management system based on the community. University of electronic science and technology, 2012.

[3] Sun Qing-xia. The application of mobile office system in the property management enterprise. Computers and telecommunications, 2013:46-48.

[4] Wu Jing. The design and implementation of remote monitoring system for the Android mobile terminal [D]. Shanghai Jiao tong University, 2012.

[5] Wang Da-lei. The design and implementation of the Network television system based on Android [D]. South China University of technology, 2012.

[6] Wang Ya-ping. The application design of Chinese voice message based on Android [D]. Inner Mongolia University of science and technology, 2012.

[7] Zhang Xin, Wang Yi. The design and implementation of a smart phone location monitoring query system [C]. 2012 national wireless and mobile communication academic conference proceedings,2012.

[8] He Xin-Qiang. The design and implementation of intelligent community management system based on the 3g wireless network. University of electronic science and technology, 2012.

[9] Guo Jia-Bei. The application software development of video monitoring based on the Android smart phone [D]. Hangzhou Electronic science and Technology University, 2013.

[10] Song Xian-Song. The design and implementation of intelligent bus system based on the Android platform. M.S [D]. Beijing University of posts and telecommunications, 2013.

[11] K. Kear, F. Chetwynd, J. Williams, H. Donelan, "Web conferencing for synchronous online tutorials," Perspectives of tutors using a new medium. Contents lists available at SciVerse 
ScienceDirect Computers \& Education. 